\title{
Lost in Time: Temporal Monitoring Elicits Clinical Decrements in Sustained Attention Post-Stroke
}

\author{
M.B. Brosnan ${ }^{1,2,3,6, *}$ (D) , P.M. Dockree ${ }^{1}$, S. Harty ${ }^{1,2}$, D.J. Pearce ${ }^{6}$, J.M. Levenstein ${ }^{2,4}$, C.R. Gillebert ${ }^{3,5}$, M.A. Bellgrove ${ }^{6}$, \\ R.G. O'Connell ${ }^{1,6}$, I.H. Robertson ${ }^{1}$ and N. Demeyere ${ }^{2}$ (D) \\ ${ }^{1}$ Trinity College Institute of Neuroscience and School of Psychology, College Green, Dublin 2, D02 PN40, Ireland \\ ${ }^{2}$ Department of Experimental Psychology, University of Oxford, Anna Watts Building, Woodstock Road, Oxford, OX2 6GG, UK \\ ${ }^{3}$ Oxford Centre for Human Brain Activity, Wellcome Centre for Integrative Neuroimaging, University of Oxford, Univesity Department of Psychiatry, \\ Warneford Hospital, Oxford, OX3 7JX, UK \\ ${ }^{4}$ Section on Functional Imaging Methods, Laboratory of Brain and Cognition, National Institutes of Mental Health, Bethesda, MD 20892, USA \\ ${ }^{5}$ Department of Brain and Cognition, KU Leuven, 3000 Leuven, Belgium \\ ${ }^{6}$ School of Psychological Sciences and Turner Institute for Brain and Mental Health, Monash University, Clayton, Melbourne, VIC 3800, Australia
}

(Received December 15, 2020; Final Revision January 18, 2021; Accepted January 29, 2021; First Published Online March 22, 2021)

\begin{abstract}
Objectives: Mental fatigue, 'brain fog', and difficulties maintaining engagement are commonly reported issues in a range of neurological and psychiatric conditions. Traditional sustained attention tasks commonly measure this capacity as the ability to detect target stimuli based on sensory features in the auditory or visual domains. However, with this approach, discrete target stimuli may exogenously capture attention to aid detection, thereby masking deficits in the ability to endogenously sustain attention over time. Methods: To address this, we developed the Continuous Temporal Expectancy Task (CTET) where individuals continuously monitor a stream of patterned stimuli alternating at a fixed temporal interval $(690 \mathrm{~ms})$ and detect an infrequently occurring target stimulus defined by a prolonged temporal duration (1020 ms or longer). As such, sensory properties of target and non-target stimuli are perceptually identical and differ only in temporal duration. Using the CTET, we assessed stroke survivors with unilateral right hemisphere damage $(N=14)$, a cohort in which sustained attention deficits have been extensively reported. Results: Stroke survivors had overall lower target detection accuracy compared with neurologically healthy age-matched older controls $(N=18)$. Critically, stroke survivors performance was characterised by significantly steeper within-block performance decrements, which occurred within short temporal windows $(\sim 31 / 2 \mathrm{~min})$, and were restored by the break periods between blocks. Conclusions: These findings suggest that continuous temporal monitoring taxes sustained attention processes to capture clinical deficits in this capacity over time, and outline a precise measure of the endogenous processes hypothesised to underpin sustained attention deficits following right hemisphere stroke.
\end{abstract}

Keywords: Stroke, Sustained Attention, Right Hemisphere, Mental Fatigue, Clinical Assessment, Attention

\section{INTRODUCTION}

Difficulties concentrating and maintaining engagement, mental fatigue, and 'brain fog' are commonly reported in a wide range of psychiatric, neurodevelopmental, and neurological conditions, including depression (Deng, Li, \& Tang, 2014), attention deficit hyperactivity disorder (ADHD) (American Psychiatric Association, 2013) stroke (Rueckert \& Grafman, 1996), Alzheimer's disease (Perry, Watson, \& Hodges, 2000), and, most recently, following COVID-19

*Correspondence and reprint requests to: MB Brosnan, Oxford Centre for Human Brain Activity, Wellcome Centre for Integrative Neuroimaging, University of Oxford, Oxford, UK. E-mail: meadhbh. brosnan@psy.ox.ac.uk
(Zhou et al., 2020). Deficits in this fundamental facet of cognition can hinder recovery and exacerbate neurocognitive issues in at least two ways. Firstly, lapses in sustained attention compromise the ability to engage with the task at hand (Smilek, Carriere, \& Cheyne, 2010). Therefore, sustained attention deficits limit the extent to which patients can engage with and accordingly benefit from learning, intervention, and rehabilitation programmes (e.g., Bennett et al., 2002; Blancgarin, 1994; Robertson, Ridgeway, Greenfield, \& Parr, 1997; van Zandvoort, Kessels, Nys, de Haan, \& Kappelle, 2005). Second, the deployment of attention is known to be critical for gating plasticity processes (Polley, Steinberg, \& Merzenich, 2006; Recanzone, Schreiner, \& Merzenich, 1993). Plasticity processes in turn underlie cortical 
remapping and functional reorganisation of the brain that are characteristic of recovery (Di Lazzaro et al., 2010; Fu \& Zuo, 2011; Hallett, 2001; Phillips, Batten, Tremblay, Aldosary, \& Blier, 2015; Qian et al., n.d.). Thus, sustained attention deficits may also hinder the extent to which functional restoration can occur at the neural level.

Several studies in neurologically healthy participants have suggested that sustained attention is supported by a predominantly right-lateralised network (Johannsen et al., 1997; Langner \& Eickhoff, 2013; Singh-Curry \& Husain, 2009; Warm, Richter, Sprague, Porter, \& Schumsky, 1980; Whitehead, 1991), modulated by the locus coeruleus norepinephrine noradrenergic arousal system (Corbetta \& Shulman, 2002; 2011; Posner \& Petersen, 1990). In correspondence with findings highlighting a privileged role for the right hemisphere $(\mathrm{RH})$ for sustained attention, stroke survivors with damage to RH networks show pronounced deficits in this capacity (Malhotra, Coulthard, \& Husain, 2009; Robertson, Manly, Beschin, \& Daini, 1997; Rueckert \& Grafman, 1996; 1998; Wilkins, Shallice, \& McCarthy, 1987), typically defined by an overall deficit in the ability to detect infrequently occurring visual targets when monitoring a continuous stream of stimuli.

Early experimental tasks designed to capture sustained attention usually asked participants to monitor targets over tens of minutes. This would typically elicit performance decrements (so-called time-on-task effects) in healthy individuals (Mackworth \& Taylor, 1963; Mackworth, 1948; Parasuraman, 1979). Subsequent assessments defined sustained attention as the ability to 'self-sustain mindful, conscious processing of stimuli' in order to capture transient lapses in attention while performing more complex, goaldirected tasks of much shorter durations (e.g., the Sustained Attention to Response Test (Manly et al., 2003; Robertson, Manly, Andrade, Baddeley, \& Yiend, 1997)). Counter-intuitively, when neurological healthy individuals perform such tasks for protracted periods of time, performance improvements as opposed to decrements are observed (Staub, Doignon-Camus, Marques-Carneiro, Bacon, \& Bonnefond, 2015; Brosnan et al., 2018). Moreover, experimental tasks assessing time on task effects in RH stroke often fail to show performance decrements over relatively short task durations ( 8-10 min; (Malhotra et al., 2009; Rueckert \& Grafman, 1996; 1998). This suggests that either RH stroke survivors do not show linear decrements in performance over time on these tasks (which is often at odds with anecdotal reports from both RH stroke survivors and their clinicians), or that sustained attention tasks may not be sufficiently sensitive to capture precise deficits in the temporal dynamics of sustained attention difficulties.

One limitation of traditional sustained attention tasks is that the discrete onset of sensory target stimuli (i.e., of either visual or auditory targets) may exogenously capture attention and inadvertently 'support' sustained attention, such that successful performance on the task is not uniquely contingent on endogenous deployment of attentional control. Moreover, the continuous mapping of a transiently presented target stimulus (e.g., number 3 from a stream of nine numbers; Manly et al., 2003; Robertson, Manly, Andrade, Baddeley, \& Yiend, 1997) with a specific behaviour (respond or withhold a response) may result in automatic detection which is characteristic of consistent-mapping paradigms (Shiffrin \& Schneider, 1977). Thus, these tasks may fail to identify decrements in attentional engagement occurring over relatively shorter timescales.

The current study employed the Continuous Temporal Expectancy Task (CTET; (O'Connell et al., 2009) in which targets and non-targets differ only in temporal duration and are otherwise perceptually identical. This eliminates any potential for exogenous attentional capture by perceptual features of the stimuli, since targets and non-targets differ only in temporal duration. Moreover, previous work has shown that with mind-wandering, time 'contracts' (Terhune, Croucher, Marcusson-Clavertz, \& Macdonald, 2017); therefore, a task necessitating the detection of relatively longer temporal intervals might be particularly sensitive to states of inattention. In the CTET, participants monitor a continuous stream of patterned stimuli that are presented centrally and make a button press when they detect a longer-duration stimulus. This task is sensitive to withinblock performance decrements (over approximately $3 \mathrm{~min}$ windows) in neurologically healthy individuals and here we assess its potential to elicit performance decrements in RH stroke survivors, a group who are clinically and experimentally characterised by sustained attention deficits (Malhotra et al., 2009; Molenberghs, Gillebert, Schoofs, \& Dupont, 2009; Robertson, Ridgeway, Greenfield, \& Parr, 1997; Rueckert \& Grafman, 1996; 1998; Wilkins et al., 1987). We reasoned that the CTET will maximally tax sustained attention in RH stroke survivors and reveal performance decrements over short time windows.

\section{METHODS}

\section{Participants}

Fourteen chronic stroke survivors with unilateral damage to the RH were recruited through an existing database of patients (either in acute settings or through self-referrals) interested in research participation at the Cognitive Neuropsychology Centre, Department of Experimental Psychology, Oxford University. All patients were $>1$ year post-stroke $(M=2.15$ years, $S D=1.07$; note these data were not available for one self-referred patient). All study participants provided written informed consent, in compliance with relevant protocols approved by the University of Oxford Central University Research Ethics Committee, and the experimental procedures were conducted in accordance with the latest version of the Declaration of Helsinki. Eighteen neurologically healthy older adults were recruited through an existing database of healthy older adults interested in participating in research at the Turner Institute for Brain and Mental Health, Monash University, Australia. The 
Table 1. Demographic information for the healthy older adults and RH stroke survivors

\begin{tabular}{lcc}
\hline \hline & Controls $(N=18)$ & Patients $(N=14)$ \\
\hline \multirow{2}{*}{ Age (years) } & $74.78(5.99)$ & $70.71(7.32)$ \\
Gender & $60-82$ & $58-84$ \\
& 11 male & 9 male \\
Education (years)* & $(47.83 \%)$ & $(64.29 \%)$ \\
& $16.89(3.45)$ & $13.23(3.40)$ \\
& $10-22$ & $8-21$ \\
\hline \hline
\end{tabular}

Note. Years in education was not available for one stroke participant.

*A significant difference between patients and controls values denote mean (SD).

experimental protocol was approved by Monash University's Human Research Ethics Committee before testing and was carried out in accordance with the approved guidelines.

\section{Demographics}

All participants provided their age, gender, and level of education (Table 1). Differences in demographic factors between healthy older adults and the stroke survivors were assessed using one way between-subject ANOVAs with 'Group' as the between-subject factor. There was no evidence to suggest that the stroke survivors and healthy older adults differed in age $\left(F_{1,30}=2.99 ; p=.09\right.$, Table 1$)$. In an effort to ensure that our results were generalisable to both genders, we aspired to obtain an equal number of male and female participants in both groups. Binomial tests conducted separately for the healthy older controls and the stroke survivors indicated that the proportion of male to female participants did not differ significantly from the expected $.50(p>.41$ for both groups, Table 1). The RH stroke survivors had spent fewer years in formal education $\left(F_{1,30}=8.98, p=.005\right.$, $\eta_{p}{ }^{2}=.23$, Table 1$)$.

\section{Lesions}

Magnetic resonance images (MRIs) were acquired on a 3-T TIM Trio scanner at the Oxford Centre for Clinical Magnetic Resonance Research (OCMR) for $N=7$ patients. These scans were acquired at the chronic stage (i.e., $>6$ months poststroke onset). High-resolution 3D whole-brain T1-weighted MRI scans were acquired using a magnetisation-prepared rapid gradient-echo sequence (TR $3000 \mathrm{~ms}$, TE $4.7 \mathrm{~ms}$, flip angle $8^{\circ}, 1 \mathrm{~mm}$ isotropic resolution) and fluid rapid attenuated inversion recovery images were also obtained acquired (TR $5000 \mathrm{~ms}$, TE 397, in-plane resolution $1 \mathrm{~mm}$, slice thickness $1.5 \mathrm{~mm})$. For the remaining patients $(n=7)$, clinical imaging was acquired at the acute stage (i.e., $<3$ weeks post-stroke onset) as part of their hospital admission diagnostics. Of these patients, lesions were delineated using either a clinical T1-weighted MRI $(n=1$, voxel resolution $1.78 \mathrm{~mm} \times 1.78 \mathrm{~mm} \times 1 \mathrm{~mm}, 192$ axial slices) or clinical

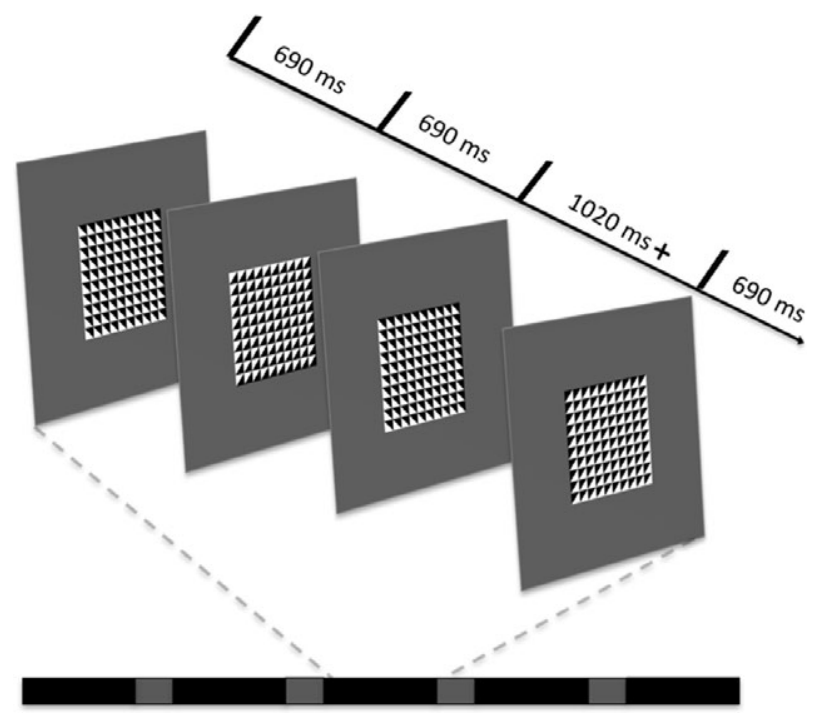

Fig. 1. The Continuous Temporal Expectancy Task (CTET). Participants monitored an alternating patterned stimulus. The visual stimulus usually alternated at a fixed temporal interval $(690 \mathrm{~ms})$ and the participants' task was to identify stimuli that were presented for a longer interval $(1020 \mathrm{~ms})$, thereby necessitating target detection in the temporal as opposed to visual domain.

computerised tomography (CT) $(n=6$, slice thickness $5 \mathrm{~mm}$, 34.17 (2.40; range 30-36) axial slices). Lesions were manually delineated in native structural space slice by slice in the axial plane (MRIcron; McCausland Center for Brain Imaging, Columbia, SC, USA). Lesion masks were smoothed at $5 \mathrm{~mm}$ full width at half maximum in the $z$-direction and binarised using a .5 intensity threshold. Native space structural scans and lesion masks were aligned to an Montreal Neurological Institute (MNI) equivalent stereotactic space $\left(2 \mathrm{~mm}^{3}\right)$ specific for each modality and generated from a healthy elderly population. The template spaces and normalisation algorithms were performed using 'Clinical Toolbox' (Rorden et al., 2012), implemented with Statistical Parametric Mapping 8 (SPM8: Wellcome Department of Cognitive Neuroscience, University College London, UK; http://www.fil.ion.ucl.ac.uk). For two patients, lesions were not yet visible at the time of their clinically acquired acute CT scans. However, both patients' medical presentation and diagnosis indicated RH stroke. The lesion distributions are shown in Figure 2a.

\section{Assessment}

\section{The Continuous Temporal Expectancy Task (CTET)}

The CTET (O'Connell, et al., 2009), a temporal judgement task designed to elicit frequent lapses of attention was employed to measure sustained attention. In this task, a patterned stimulus was presented centrally on a grey background and was constantly rotated at $90^{\circ}$ angles (see Figure 1). The pattern stimulus consisted of a single $8 \mathrm{~cm}^{2}$ large square divided into a $10 \times 10$ grid of identical square tiles $\left(0.8 \mathrm{~mm}^{2}\right)$, 
(a)

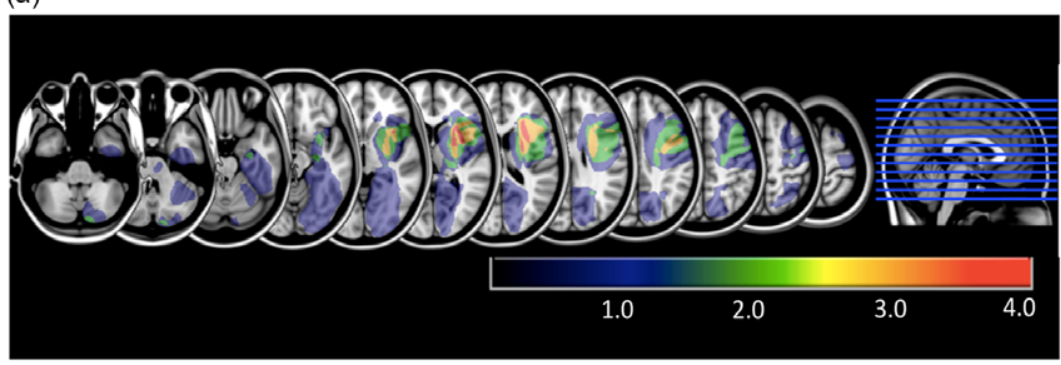

(b)

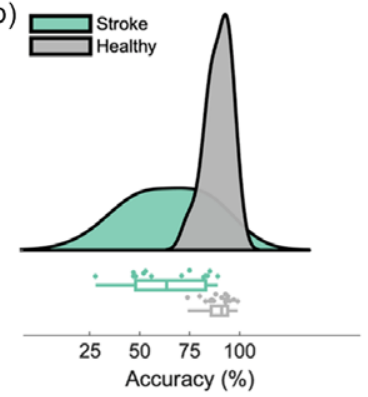

(c)

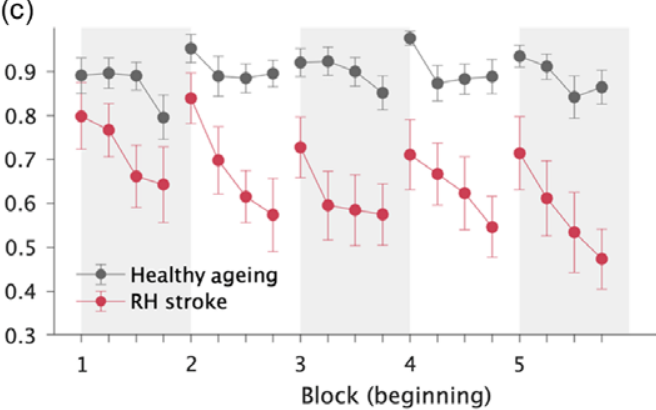

Fig. 2. (a) Lesion overlay for the right hemisphere stroke survivors. Conjunction maps were created from each patients' delineated lesion mask $(N=12)$ registered to a template space (MNI152 T1 $1 \mathrm{~mm})$. Intensity values represent the number of subjects with overlapping lesion affected anatomy. (b) Overall accuracy levels for stroke survivors and neurological healthy older adults. In line with previous sustained attention studies, patients with unilateral RH damage were less accurate on the CTET as compared with neurologically healthy older adults. (c) Performance decrements over time on the CTET. Performance decrements within the duration of the task blocks were significantly steeper for the patient cohort relative to the neurologically healthy older adults. Note: alternating grey and white columns depict the start of each task block.

each one diagonally split into black and white halves. The tile orientation shifted by $90^{\circ}$ in a random direction (clockwise or counterclockwise) on each frame change, yielding four distinct patterns. In the 'standard' trials ( $\sim 90 \%$ of trials), the stimuli were presented for a temporal duration of $690 \mathrm{~ms}$. The participant's task was to identify the infrequent 'target' trials by a button press using their right index finger, where the stimulus was presented for a longer temporal duration. Stimuli were pseudo-randomly presented such that there were between 7 and 15 (average of 11) standard trials between each target presentation.

The CTET was designed such that temporal judgements were perceptually undemanding for participants but challenging when tasked to continuously perform the judgements over sustained periods of time. Prior to the experimental blocks, a practice block was administered, where all participants were required to demonstrate $100 \%$ accuracy, before advancing to the experimental blocks. For the practice block, three targets were randomly interspersed among 25 standard stimuli. Target stimuli were presented at a target duration of $1020 \mathrm{~ms}$, that is, $330 \mathrm{~ms} / 47.83 \%$ longer than standard trials. If participants missed one or more target stimuli, the block was performed again. If the participant still failed to identify all the targets, the duration of the target stimulus was adapted such that all participants demonstrated $100 \%$ accuracy on two consecutive blocks. For the healthy older adults, all participants identified the target stimuli at $100 \%$ accuracy during the practice blocks at the target duration of $1020 \mathrm{~ms}$. Of the stroke survivors, seven identified the target stimulus at a duration of $1020 \mathrm{~ms}$ at $100 \%$ accuracy. For the other seven patients, the duration of the target stimulus was adapted as follows such that all participants demonstrated $100 \%$ accuracy on two consecutive practice blocks: if the patient did not achieve 100\% accuracy during two consecutive blocks within the first three practice blocks, the duration of the target stimulus was increased to $1140 \mathrm{~ms}$. Participants were then given two practice blocks with this target duration. If $100 \%$ accuracy was achieved during these blocks, then this target duration was set at $1140 \mathrm{~ms}$ for the rest of the experiment. If the patient did not exhibit $100 \%$ accuracy at this duration, the target duration was subsequently increased again to $1500 \mathrm{~ms}$, and following this to $1800 \mathrm{~ms}$. In order to ensure the stroke survivors fully understood the task requirements, all patients were required to complete two consecutive practice blocks at $100 \%$ accuracy before proceeding to the experimental trials. For the experimental trials, the target durations for the stroke survivors were either $1020 \mathrm{~ms}(N=7), 1140 \mathrm{~ms}(N=2)$, $1500 \mathrm{~ms}(N=4)$, or $1800 \mathrm{~ms}(N=1)$.

This tailored approach was adopted to ensure that any observed differences in attention could not be attributed to the perceptual demands of the task. Participants completed five blocks of the task and were given a break $(\sim 1 \mathrm{~min})$ in between all blocks. Each block consisted of 225 stimulus rotations with a total duration of between $3 \mathrm{~min}$ and $5 \mathrm{~s}$ and $3 \mathrm{~min}$ and $30 \mathrm{~s}$. The number of targets varied between 18 and 22 per block. 


\section{Analyses}

Sustained attention was expressed as the percentage of correct target detections. A target was considered correctly identified if a participant responded within 2.07 seconds (i.e., three standard trials) of a target trial. One stroke patient presented with working memory problems, identified by a previous study in the centre using the Oxford Cognitive Screen (Demeyere, Riddoch, Slavkova, Bickerton, \& Humphreys, 2015). In an effort to exclude the possibility that these deficits were contributing to performance on the task, this patient was required to recall the task instructions at the end of each block. Outliers were defined as participants whose overall accuracy levels were greater than three times the interquartile range, which was determined separately for the healthy older adults and stroke survivors.

We examined overall accuracy across all blocks of the task (Figure 2b), and we also evaluated changes in accuracy both within each block and across blocks (Figure 2c). Differences in accuracy between the stroke survivors and healthy older adults were evaluated using a repeatedmeasures mixed ANOVA with 'Performance Decrement Within Blocks' (within four quartiles of each block) and 'Performance Decrement Across Blocks' (five blocks) as within subject factors. 'Group' (RH stroke, healthy ageing) was included as a between-subject factor. In order to assess the putatively restorative effect of task break on performance, the average of the fourth quartile computed across blocks $1,2,3$, and 4 was compared against the average of the first quartile across blocks 2, 3, 4, and 5 using a repeated-measures ANOVA with break as a within-subjects and group as a between-subjects factor.

The verify that any of the observed effects could not be attributed to group differences in levels of formal eduction, all significant ANOVAs were repeated years in formal education included as a covariate.

\section{RESULTS}

\section{Overall Target Detection (Accuracy)}

The rate of false alarms (i.e., the number of button presses in the absence of a target stimulus) was low on the CTET $(M=3.36, S D=2.03$, range $=1.2-9.4 \%)$ indicating that all participants were performing well above chance level and had correctly understood the instructions. In line with previous reports investigating overall performance levels in RH stroke survivors (Robertson, Manly, Andrade, Baddeley, \& Yiend, 1997; Rueckert \& Grafman, 1996; 1998), a main effect of Group indicated that the patients were significantly less accurate at identifying target stimuli, compared with the healthy older controls $\left(F_{1,29}=25.34\right.$, $p<.0005, \eta_{p}{ }^{2}=.47$, Figure $2 \mathrm{~b}$; RH stroke M(SD) $64.27 \%$ (19.17); healthy older controls $\mathrm{M}(\mathrm{SD}) 89.29 \%$ (6.56)). This main effect remained significant after controlling for years of education $\left(F_{1,28}=16.70, p<.0005, \eta p^{2}=.37\right)$.
Time on Task Decrements: Performance Decrement Within Blocks

A main effect of Quartile $\left(F_{3,87}=15.58, p<.0005, \eta_{p}{ }^{2}=.35\right)$ demonstrated that accuracy on the task decreased within each block (see Figure 2c), as confirmed by a significant linear contrast $\left(F_{1,29}=42.36, p<.0005, \eta_{p}{ }^{2}=.59\right)$. Critically, this was qualified by a Group $\times$ Quartile interaction $\left(F_{3,87}=3.21\right.$, $\left.p=.03, \eta_{p}{ }^{2}=.10\right)$. Follow-up repeated-measures ANOVAs with Quartile as the within-subjects factor were conducted separately for the neurologically healthy older group and the RH stroke survivors. For both the healthy group $\left(F_{3,48}=3.58, p=.02, \eta_{p}{ }^{2}=.18\right)$ and the stroke survivors $\left(F_{3,39}=11.19, p<.0005, \eta_{p}{ }^{2}=.46\right)$, there was a main effect of Quartile, best captured by a linear fit (healthy; $F_{1,16}=7.41$, $p=.02, \eta_{p}{ }^{2}=.32$; stroke: $F_{1,13}=38.37, p<.0005, \eta_{p}{ }^{2}=.75$ ). Thus, the significant Group $\times$ Quartile interaction was driven by a significantly steeper within-block decrement in the accuracy of the RH stroke survivors relative to the healthy older controls. When controlling for years of education, the main effect of Quartile was no longer significant $\left(F_{3,84}=1.14\right.$, $\mathrm{p}=.34)$. Crucially, however, the Group $\times$ Quartile interaction remained significant $\left(F_{3,84}=3.73, \mathrm{p}=.01, \eta_{p}{ }^{2}=.12\right)$.

\section{Time on Task Decrements: Performance Decrement Across Blocks}

There was no main effect of Block $\left(F_{4,116}=1.78, p=.14\right)$, but there was a Group $\times$ Block interaction $\left(F_{4,116}=2.75\right.$, $\left.\mathrm{p}=.03, \eta_{p}{ }^{2}=.09\right)$ which remained significant after controlling for education $\left(F_{4,112}=2.66, \mathrm{p}=.04, \eta_{p}{ }^{2}=.09\right)$ indicating that the two groups differed in the way their performance changed across blocks. Follow-up repeated-measures analyses conducted for each group separately failed to resolve the source of this interaction with no main effect of Block for either the stroke $\left(F_{4,52}=2.31, p=.07, \eta_{p}{ }^{2}=.15\right)$ or the healthy older cohort $\left(F_{4,64}=.85, p=.5\right)$. As such, these results suggest that while the stroke survivors exhibit particularly sharp decrements in their ability to sustain attention within blocks, we found no evidence to suggest that the stroke survivors differed from controls in their capacity to sustain attentional engagement over coarser timescales (i.e., across blocks). Finally, there were no other significant interaction terms $($ Quartile $\times$ Block, and Quartile $\times$ Block $\times$ Group both $p>.72)$.

\section{Break Periods to Restore Performance}

The findings thus far indicate that deficits in attention arise from difficulties in the capacity to maintain engagement over short time periods (within block). This suggests that the short break periods in between blocks exerted a restorative effect over performance. Formal analyses supported this hypothesis. A main effect of Break was observed $\left(F_{1,29}=36.76\right.$, $\left.p<.0005, \eta_{p}{ }^{2}=.56\right)$, and there was no significant Break $\times$ Group interaction effect $\left(F_{1,29}=3.30, p=.08\right.$, 
$\eta_{p}{ }^{2}=.10$ ). As such, for both the healthy older adults and the RH stroke survivors, performance during the fourth quartiles (healthy older $M=85.83 \%, S D=12.07$, RH stroke $M=58.45 \%, S D=23.78$ ) was significantly lower than during the first quartile (healthy older $M=94.63 \%, S D=7.68$; RH stroke $M=74.79 \%, S D=21.23$ ), demonstrating that irrespective of group, the performance deficit was offset by rest periods between blocks.

\section{DISCUSSION}

Here we show that monitoring attention in the temporal domain captures clinical deficits in maintaining attention within a shorter time window (a linear decline in performance within $\sim 3 \frac{1}{2} \mathrm{~min}$ ) than previously noted with $\mathrm{RH}$ stroke survivors (Robertson, Ridgeway, Greenfield, \& Parr, 1997; Rueckert \& Grafman, 1996; 1998). Our results indicate that well-established clinical deficits in sustained attention (overall target detection accuracy) following RH stroke (Robertson et al., 1997; Rueckert \& Grafman, 1996; 1998) may arise from a specific impairment in the ability to maintain engagement over relatively short temporal windows. As such, these results suggest that the CTET (O'Connell, et al., 2009) is a sensitive tool for capturing and examining performance decrements in sustained attention over short temporal windows.

There are several aspects of the CTET that make it appealing for assessing attentional deficits, particularly in patient populations. Firstly, the target stimulus is adapted such that $100 \%$ accuracy is exhibited during the practice trials preceding the experimental blocks. Thus, the task is not confounded by an overall perceptual deficit in the stroke survivors. Secondly, unlike many sustained attention tasks (e.g., Malhotra et al., 2009; Manly et al., 2003; Rueckert \& Grafman, 1996; 1998), identification of the target stimulus in the CTET is facilitated by continuously monitoring time as opposed identifying distinct perceptual characteristics of targets relative to non-targets. This is potentially particularly advantageous in producing a performance decrement as visual search processes which may help with the identification of target stimuli, such as stimulus-driven attentional capture and stimulus-response mapping, are reduced in the CTET, thus placing greater demands on the continuous monitoring process and taxing the sustained attention system.

Despite the stroke survivors demonstrating steep withinblock performance declines, they also exhibited the capacity to temporarily revive their sustained attention following the brief within-block rest periods, an observation supported by significantly higher performance during the first quartiles of blocks 2, 3, 4, and 5, as compared with the last quartiles of blocks 1, 2, 3, and 4. In the current study, the within-block performance decrement observed in the RH stroke survivors did not get worse with time (i.e., there was no interaction between this effect and the overall performance decrement over the course of the experiment). This suggests that the sustained attention system can be revived within intervals as short as the between block breaks of approximately $1 \mathrm{~min}$.
This is in line with work showing that sustained attention can be modulated by gaining volitional control over biophysiological markers of arousal (O'Connell, Bellgrove, Dockree, \& Lau, 2008; Robertson, Tegnér, Tham, \& Lo, 1995) or by presenting salient sensory stimuli which act as external alerting cues (Finke, Matthias, Keller, Müller, \& Schneider, 2012; Robertson, Mattingley, Rorden, \& Driver, 1998).

In this study, we observed large interindividual differences in sustained attention capacity on the CTET, particularly in the patient cohort. An avenue for future work will be to highlight neural sources of variability on the CTET along with the functional, neuropsychological, and clinical implications of deficits in the capacity to sustain engagement. While our study highlights the feasibility of the CTET to effectively elicit sustained attention deficits in a relatively small cohort that is characterised by known difficulties in this capacity, future confirmation of our results will be required in larger-scale clinical studies. In particular, it will be important to assess whether the CTET is sensitive to attention deficits following exclusively right hemisphere infarcts or whether this task is sensitive to other neurological events, including brainstem damage, left hemisphere lesions, traumatic brain injury, and neuroinflammation. Moreover, our groups differed both in terms of geographical location and levels of educational attainment, and future research should address whether cultural, environmental, and cognitively enriching factors including education may mitigate deficits in the capacity to sustain engagement.

One likely explanation for these sharp decreases in arousal following RH damage is dysfunction of the noradrenergic system. Noradrenaline (norepinephrine), a neurotransmitter emanating from the locus coeruleus with widespread projections throughout the cortex, plays a well-established role in maintaining arousal (Corbetta \& Shulman, 2002; 2011; Posner \& Petersen, 1990), for a review see Sara, 2009. $\mathrm{RH}$ dominance of noradrenaline is evident from asymmetric organisation of the noradrenergic system in rats (Robinson, 1979; Robinson \& Coyle, 1980), higher noradrenergic concentrations in the right thalamus in post-mortem human neurochemical studies (Oke, Keller, Mefford, \& Adams, 1978), and increased blood-oxygen-level-dependent imaging (BOLD) activation exclusively within the RH during pharmacological upregulation of noradrenaline (Grefkes, Wang, Eickhoff, \& Fink, 2010). Pharmacological interventions such as noradrenergic agonists and methylphenidate that modulate noradrenergic levels within prefrontal regions have been shown to improve sustained attention performance on the CTET in healthy younger adults (Dockree et al., 2017), and preliminary evidence suggests that this may be a useful approach to improve sustained attention deficits associated with damage to the RH (Malhotra, Parton, Greenwood, \& Husain, 2006; Singh-Curry, Malhotra, Farmer, \& Husain, 2011). Future work should directly explore whether noradrenergic dysfunction underlies the pronounced decrements in sustained attention observed in the current study, and whether manipulating activity within this right-lateralised system, for 
example, through pharmacological interventions (Dockree et al., 2017), behavioural training paradigms (MilewskiLopez et al., 2014), functional Magnetic Resonance Imaging (fMRI) neurofeedback training techniques (deBettencourt, Cohen, Lee, Norman, \& Turk-Browne, 2015; Habes et al., 2016), or brain stimulation (Brosnan et al., 2017; 2018), might ameliorate these performance decrements.

Finally, although specific functional impairments resulting from a stroke have often been considered in the context of modular damage (e.g., Broca, 1865), there is a growing consensus that it is not only damage to specific regions but rather disruptions to inter-connected neural networks which are associated with functional impairments (e.g., Forkel et al., 2014; cf Baldassarre, Ramsey, Siegel, Shulman, \& Corbetta, 2016; Corbetta \& Shulman, 2011). With regard to the sustained attention system, recent multivariate analyses of fMRI data have identified a widespread network of cortical, subcortical, and cerebellar regions underlying the capacity to maintain attentional engagement (deBettencourt et al., 2015; Rosenberg et al., 2016). Rosenberg and colleagues recently showed that only $27 \%$ of a multivariate pattern analysis-derived network which successfully predicted the 'high' attention state both in healthy younger adults and children and adults with attentional deficits (ADHD symptoms) included prefrontal and parietal nodes. These findings do not discount fronto-parietal networks contributions to maintaining sustained attention (Rueckert \& Grafman, 1996; 1998; Wilkins et al., 1987) but rather demonstrate the role of these regions within a widespread network (Shalev, Humphreys, \& Demeyere, 2016). The heterogeneity of the lesions in the current cohort support these findings and suggest damage to widespread cortical regions within the right-lateralised noradrenergic network results in deficits maintaining sustained attention over time (cf, (Corbetta \& Shulman, 2011).

The current findings hold important translational implication for informing cognitive assessment and rehabilitation strategies for individuals who have experienced RH stroke, and in particular, they speak to the need for employing assays that are sensitive to detecting deficits over short time intervals when appraising the integrity of sustained attention in this cohort.

\section{ACKNOWLEDGEMENTS}

We would like to sincerely thank the late Prof Glyn Humphreys for his valuable contributions to the design and implementation of this project. We wish to thank Tara Anne Maguire for her assistance with data collection, and our participants in Melbourne and Oxford for generously volunteering their time. This work was supported by the European Union FP7 Marie Curie Initial Training Network Individualised Diagnostics \& Rehabilitation of Attention Disorders (grant number 606901), a Marie Skłodowska-
Curie Fellowship from the European Commission (AGEING PLASTICITY; grant number 844246), and supported by the NIHR Oxford Health Biomedical Research Centre. The Wellcome Centre for Integrative Neuroimaging is supported by core funding from the Wellcome Trust (203139/Z/16/Z). CR Gillebert was supported by an Odysseus grant of the Research Foundation Flanders (G0H7718N). Paul Dockree was supported by an Irish Research Council Laureate grant: IRCLA/2017/306.

\section{CONFLICTS OF INTEREST}

The authors have nothing to disclose.

\section{REFERENCES}

American Psychiatric Association (2013). Diagnostic and statistical manual of mental disorders, Fifth edn. Arlington, VA: American Psychiatric Association.

Baldassarre, A., Ramsey, L.E., Siegel, J.S., Shulman, G.L., \& Corbetta, M. (2016). Brain connectivity and neurological disorders after stroke. Current Opinion in Neurology, 29(6), 706-713. http://doi.org/10.1097/WCO.0000000000000396

Bennett, H.P., Corbett, A.J., Gaden, S., Grayson, D.A., Kril, J.J., \& Broe, G.A. (2002). Subcortical vascular disease and functional decline: a 6-year predictor study. Journal of the American Geriatrics Society, 50(12), 1969-1977. http://doi.org/10.1046/j. 1532-5415.2002.50608.x

Blanc-garin, J. (1994). Patterns of recovery from hemiplegia following stroke. Neuropsychological Rehabilitation, 4(4), 359-385. http://doi.org/10.1080/09602019408401606

Broca, P. (1865). Sur le siège de la faculté du langage articulé. Bulletins Et Mémoires De La Société d'Anthropologie De Paris, 377-393.

Brosnan, M.B., Arvaneh, M., Harty, S., Maguire, T., O'Connell, R., Robertson, I.H., \& Dockree, P.M. (2018). Prefrontal modulation of visual processing and sustained attention in aging, a transcranial direct current stimulation-electroencephalogram coregistration approach. Journal of Cognitive Neuroscience. http://doi. org/10.1162/jocn_a_01307

Brosnan, M.B., Demaria, G., Petersen, A., Dockree, P.M., Robertson, I.H., \& Wiegand, I. (2017). Plasticity of the rightlateralized cognitive reserve network in ageing. Cerebral Cortex, 28(5), 1749-1759. http://doi.org/10.1093/cercor/bhx085

Corbetta, M. \& Shulman, G.L. (2002). Control of goal-directed and stimulus-driven attention in the brain. Nature Reviews Neuroscience, 3(3), 215-229. http://doi.org/10.1038/nrn755

Corbetta, M. \& Shulman, G.L. (2011). Spatial neglect and attention networks. Annual Review of Neuroscience, 34(1), 569-599. http://doi.org/10.1146/annurev-neuro-061010-113731

deBettencourt, M.T., Cohen, J.D., Lee, R.F., Norman, K.A., \& Turk-Browne, N.B. (2015). Closed-loop training of attention with real-time brain imaging. Nature Neuroscience, 18(3), 470-475. http://doi.org/10.1038/nn.3940

Demeyere, N., Riddoch, M.J., Slavkova, E.D., Bickerton, W.-L., \& Humphreys, G.W. (2015). The Oxford Cognitive Screen (OCS): validation of a stroke-specific short cognitive screening tool. Psychological Assessment, 27(3), 883-894. http://doi.org/10. 1037/pas0000082 
Deng, Y.Q., Li, S., \& Tang, Y.Y. (2014). The relationship between wandering mind, depression and mindfulness. Vol. 5, (pp. 124-128). Mindfulness. http://doi.org/10.1007/s12671-012-0157-7.pdf

Di Lazzaro, V., Profice, P., Pilato, F., Capone, F., Ranieri, F., Pasqualetti, P., et al. (2010). Motor cortex plasticity predicts recovery in acute stroke. Cerebral Cortex, 20(7), 1523-1528. http://doi.org/10.1093/cercor/bhp216

Dockree, P.M., Barnes, J.J., Matthews, N., Dean, A.J., Abe, R., Nandam, L.S., et al. (2017). The effects of methylphenidate on the neural signatures of sustained attention. Biological Psychiatry. http://doi.org/10.1016/j.biopsych.2017.04.016

Finke, K., Matthias, E., Keller, I., Müller, H.J., \& Schneider, W.X. (2012). How does phasic alerting improve performance in patients with unilateral neglect? A systematic analysis of attentional processing capacity and spatial weighting .... Neuropsychologia, 50(6), 1178-1189. http://doi.org/10.1016/j. neuropsychologia.2012.02.008

Forkel, S.J., Thiebaut de Schotten, M., Dell'Acqua, F., Kalra, L., Murphy, D.G.M., Williams, S.C.R., \& Catani, M. (2014). Anatomical predictors of aphasia recovery: a tractography study of bilateral perisylvian language networks. Brain, 137(7), 2027-2039. http://doi.org/10.1093/brain/awu113

Fu, M. \& Zuo, Y. (2011). Experience-dependent structural plasticity in the cortex. Trends in Neurosciences, 34(4), 177-187. http://doi. org/10.1016/j.tins.2011.02.001

Grefkes, C., Wang, L.E., Eickhoff, S.B., \& Fink, G.R. (2010). Noradrenergic modulation of cortical networks engaged in visuomotor processing. Cerebral Cortex, 20(4), 783-797. http://doi. org/10.1093/cercor/bhp144

Habes, I., Rushton, S., Johnston, S.J., Sokunbi, M.O., Barawi, K., Brosnan, M., et al. (2016). fMRI neurofeedback of higher visual areas and perceptual biases. Neuropsychologia, 85(C), 208-215. http://doi.org/10.1016/j.neuropsychologia.2016.03.031

Hallett, M. (2001). Plasticity of the human motor cortex and recovery from stroke. Brain Research Reviews, 36(2-3), 169-174. http://doi.org/10.1016/S0165-0173(01)00092-3

Johannsen, P., Jakobsen, J., Bruhn, P., Hansen, S.B., Gee, A., Stødkilde-Jørgensen, H., \& Gjedde, A. (1997). Cortical sites of sustained and divided attention in normal elderly humans. NeuroImage, 6(3), 145-155. http://doi.org/10.1006/nimg.1997. 0292

Langner, R. \& Eickhoff, S.B. (2013). Sustaining attention to simple tasks: a meta-analytic review of the neural mechanisms of vigilant attention. Psychological Bulletin. http://doi.org/10.1037/ a0030694.supp

Mackworth, J.F. \& Taylor, M.M. (1963). The d'measure of signal detectability in vigilance-like situations. Canadian Journal of Psychology, 17, 302-325.

Mackworth, N.H. (1948). The breakdown of vigilance during prolonged visual search. Quarterly Journal of Experimental Psychology, 1(1), 6-21. http://doi.org/10.1080/1747021 4808416738

Malhotra, P., Coulthard, E.J., \& Husain, M. (2009). Role of right posterior parietal cortex in maintaining attention to spatial locations over time. Brain, 132(3), awn350-660. http://doi.org/10. 1093/brain/awn350

Malhotra, P.A., Parton, A.D., Greenwood, R., \& Husain, M. (2006). Noradrenergic modulation of space exploration in visual neglect. Annals of Neurology, 59(1), 186-190. http://doi.org/10.1002/ana. 20701

Manly, T., Owen, A.M., McAvinue, L., Datta, A., Lewis, G.H., Scott, S.K., et al. (2003). Enhancing the sensitivity of a sustained attention task to frontal damage: convergent clinical and functional imaging evidence. Neurocase, 9(4), 340-349. http://doi. org/10.1076/neur.9.4.340.15553

Milewski-Lopez, A., Greco, E., van den Berg, F., McAvinue, L.P., McGuire, S., \& Robertson, I.H. (2014). An evaluation of alertness training for older adults. Frontiers in Aging Neuroscience, 6(60), 843. http://doi.org/10.3389/fnagi.2014.00067

Molenberghs, P., Gillebert, C.R., Schoofs, H., Dupont, P., Peeters, R., \& Vandenberghe, R. (2009). Lesion neuroanatomy of the Sustained Attention to Response task. Neuropsychologia, 47(13), 2866-2875. http://doi.org/10.1016/j.neuropsychologia. 2009.06.012

O'Connell, R.G., Bellgrove, M.A., Dockree, P.M., \& Lau, A. (2008). Self-alert training: Volitional modulation of autonomic arousal improves sustained attention. Neuropsychologia, 46(5), 1379-1390. http://doi.org/10.1016/j.neuropsychologia.2007.12.018

O'Connell, R.G., Dockree, P.M., Robertson, I.H., Bellgrove, M.A., Foxe, J.J., \& Kelly, S.P. (2009). Uncovering the neural signature of lapsing attention: electrophysiological signals predict errors up to $20 \mathrm{~s}$ before they occur. Journal of Neuroscience, 29(26), 8604-8611. http://doi.org/10.1523/JNEUROSCI.596708.2009

Oke, A., Keller, R., Mefford, I., \& Adams, R.N. (1978). Lateralization of norepinephrine in human thalamus. Science, 200(4348), 1411-1413. http://doi.org/10.1126/science.663623

Parasuraman, R. (1979). Memory load and event rate control sensitivity decrements in sustained attention. Science, 205(4409), 924-927. http://doi.org/10.1126/science.472714

Perry, R.J., Watson, P., \& Hodges, J.R. (2000). The nature and staging of attention dysfunction in early (minimal and mild) Alzheimer's disease: relationship to episodic and semantic memory impairment. Neuropsychologia, 38(3), 252-271. http:// doi.org/10.1016/S0028-3932(99)00079-2

Phillips, J.L., Batten, L.A., Tremblay, P., Aldosary, F., \& Blier, P. (2015). A prospective, longitudinal study of the effect of remission on cortical thickness and hippocampal volume in patients with treatment-resistant depression. International Journal of Neuropsychopharmacology, 18(8), pyv037-pyv037. http://doi. org/10.1093/ijnp/pyv037

Polley, D.B., Steinberg, E.E., \& Merzenich, M.M. (2006). Perceptual learning directs auditory cortical map reorganization through top-down influences. The Journal of Neuroscience, 26(18), 4970-4982. http://doi.org/10.1523/JNEUROSCI.377105.2006

Posner, M.I. \& Petersen, S.E. (1990). The attention system of the human brain. Annual Review of Neuroscience, 13(1), 25-42. http://doi.org/10.1146/annurev.ne.13.030190.000325

Qian, X., Loo, B., Castellanos, F.X., Liu, S., Translational, H.K. (n.d.). Brain-computer-interface-based intervention re-normalizes brain functional network topology in children with attention deficit/hyperactivity disorder. Nature.com.

Recanzone, G.H., Schreiner, C.E., \& Merzenich, M.M. (1993). Plasticity in the frequency representation of primary auditory cortex following discrimination training in adult owl monkeys. The Journal of Neuroscience, 13(1), 87-103.

Robertson, I.H., Manly, T., Andrade, J., Baddeley, B.T., \& Yiend, J. (1997). 'Oops!': performance correlates of everyday attentional failures in traumatic brain injured and normal subjects. Neuropsychologia, 35(6), 747-758. http://doi.org/10.1016/S0 028-3932(97)00015-8

Robertson, I.H., Manly, T., Beschin, N., \& Daini, R. (1997). Auditory sustained attention is a marker of unilateral spatial 
neglect. Neuropsychologia, 35(12), 1527-1532. http://doi.org/ 10.1016/s0028-3932(97)00084-5

Robertson, I.H., Mattingley, J.B., Rorden, C., \& Driver, J. (1998). Phasic alerting of neglect patients overcomes their spatial deficit in visual awareness. Nature, 395(6698), 169-172. http://doi.org/ $10.1038 / 25993$

Robertson, I.H., Ridgeway, V., Greenfield, E., \& Parr, A. (1997). Motor recovery after stroke depends on intact sustained attention: a 2-year follow-up study. Neuropsychology, 11(2), 290.

Robertson, I.H., Tegnér, R., Tham, K., \& Lo, A. (1995). Sustained attention training for unilateral neglect: theoretical and rehabilitation implications. Journal of Clinical ..., 17(3), 416-430. http:// doi.org/10.1080/01688639508405133

Robinson, R. (1979). Differential behavioral and biochemical effects of right and left hemispheric cerebral infarction in the rat. Science, 205(4407), 707-710. http://doi.org/10.1126/science.462179

Robinson, R.G. \& Coyle, J.T. (1980). The differential effect of right versus left hemispheric cerebral infarction on catecholamines and behavior in the rat. Brain Research, 188(1), 63-78. http://doi.org/ 10.1016/0006-8993(80)90557-0

Rosenberg, M.D., Finn, E.S., Scheinost, D., Papademetris, X., Shen, X., Constable, R.T., \& Chun, M.M. (2016). A neuromarker of sustained attention from whole-brain functional connectivity. Nature Neuroscience, 19(1), 165-171. http://doi.org/10.1038/ nn. 4179

Rueckert, L. \& Grafman, J. (1996). Sustained attention deficits in pat ients with right frontal lesions. Neuropsychologia, 34(10), 953-963.

Rueckert, L. \& Grafman, J. (1998). Sustained attention deficits in patients with lesions of posterior cortex. Neuropsychologia, 36(7), 653-660.

Sara, S.J. (2009). The locus coeruleus and noradrenergic modulation of cognition. Nature Reviews Neuroscience, 10(3), 211-223. http://doi.org/10.1038/nrn2573

Shalev, N., Humphreys, G., \& Demeyere, N. (2016). Assessing the temporal aspects of attention and its correlates in aging and chronic stroke patients. Neuropsychologia, 1-10. http://doi.org/ 10.1016/j.neuropsychologia.2016.08.001

Shiffrin, R.M. \& Schneider, W. (1977). Controlled and automatic human information processing: II. Perceptual learning, automatic attending and a general theory. Psychological Review, 84(2), 127-190. http://doi.org/10.1037/0033-295x.84.2.127
Singh-Curry, V. \& Husain, M. (2009). The functional role of the inferior parietal lobe in the dorsal and ventral stream dichotomy. Neuropsychologia, 47(6), 1434-1448. http://doi.org/10.1016/j. neuropsychologia.2008.11.033

Singh-Curry, V., Malhotra, P., Farmer, S.F., \& Husain, M. (2011). Attention deficits following ADEM ameliorated by guanfacine. Journal of Neurology, Neurosurgery \& Psychiatry, 82(6), 688-690. http://doi.org/10.1136/jnnp.2009.195792

Smilek, D., Carriere, J.S.A., \& Cheyne, J.A. (2010). Failures of sustained attention in life, lab, and brain: Ecological validity of the SART. Neuropsychologia, 48(9), 2564-2570. http://doi.org/ 10.1016/j.neuropsychologia.2010.05.002

Staub, B., Doignon-Camus, N., Marques-Carneiro, J.E., Bacon, É., \& Bonnefond, A. (2015). Age-related differences in the use of automatic and controlled processes in a situation of sustained attention. Neuropsychologia, 75, 607-616. http://doi.org/10.1016/ j.neuropsychologia.2015.07.021

Terhune, D.B., Croucher, M., Marcusson-Clavertz, D., \& Macdonald, J.S.P. (2017). Time contracts and temporal precision declines when the mind wanders. Journal of Experimental Psychology: Human Perception and Performance, 43(11), 1864-1871. http://doi.org/10.1037/xhp0000461

van Zandvoort, M.J.E., Kessels, R.P.C., Nys, G.M.S., de Haan, E.H.F., \& Kappelle, L.J. (2005). Early neuropsychological evaluation in patients with ischaemic stroke provides valid information. Clinical Neurology and Neurosurgery, 107(5), 385-392. http:// doi.org/10.1016/j.clineuro.2004.10.012

Warm, J.S., Richter, D.O., Sprague, R.L., Porter, P.K., \& Schumsky, D.A. (1980). Listening with a dual brain: Hemispheric asymmetry in sustained attention. Bulletin of the Psychonomic Society, 15(4), 229-232. http://doi.org/10.3758/ BF03334516

Whitehead, R. (1991). Right hemisphere processing superiority during sustained visual attention. Journal of Cognitive Neuroscience, 3(4), 329-334. http://doi.org/10.1162/jocn.1991.3.4.329

Wilkins, A.J., Shallice, T., \& McCarthy, R. (1987). Frontal lesions and sustained attention. Neuropsychologia, 25(2), 359-365.

Zhou, H., Lu, S., Chen, J., Wei, N., Wang, D., Lyu, H., et al. (2020). The landscape of cognitive function in recovered COVID-19 patients. Journal of Psychiatric Research, 129, 98-102. http:// doi.org/10.1016/j.jpsychires.2020.06.022 